\title{
Attraction and consumption of methyl eugenol by male Bactrocera umbrosa Fabricius (Diptera: Tephritidae) promotes conspecific sexual communication and mating performance
}

\begin{abstract}
The Artocarpus fruit fly, Bactrocera umbrosa (Fabricius) (Diptera: Tephritidae), is an oligophagous fruit pest infesting Moraceae fruits, including jackfruit (Artocarpus heterophyllus Lamarck), a fruit commodity of high value in Malaysia. The scarcity of fundamental biological, physiological and ecological information on this pest, particularly in relation to behavioural response to phytochemical lures, which are instrumental to the success of many area-wide fruit fly control and management programmes, underpins the need for studies on this much-underrated pest. The positive response of $B$. umbrosa males to methyl eugenol (ME), a highly potent phytochemical lure, which attracts mainly males of many Bactrocera species, was shown to increase with increasing age. As early as 7 days after emergence (DAE), ca. $22 \%$ of males had responded to ME and over $50 \%$ by 10 DAE, despite no occurrence of matings (i.e. the males were still sexually immature). Male attraction to $\mathrm{ME}$ peaked from 10 to 27 DAE, which corresponded with the flies' attainment of sexual maturity. In wind-tunnel assays during the dusk courtship period, ME-fed males exhibited earlier calling activity and attracted a significantly higher percentage of virgin females compared with ME-deprived males. ME-fed males enjoyed a higher mating success than ME-deprived males at 1-day post ME feeding in semi-field assays. ME consumption also promotes aggregation behaviour in B. umbrosa males, as demonstrated in wind-tunnel and semi-field assays. We suggest that ME plays a prominent role in promoting sexual communication and enhancing mating performance of the Artocarpus fruit fly, a finding that is congruent with previous reports on the consequences of $\mathrm{ME}$ acquisition by other economically important Bactrocera species.
\end{abstract}

Keyword: Artocarpus fruit fly; Methyl eugenol; Attraction; Sexual maturation; Mating competitiveness 